\title{
Optimal linear Bernoulli factories for small mean problems*
}

\author{
Mark Huber \\ mhuber@cmc.edu \\ Version: September 29, 2016
}

\begin{abstract}
Suppose a coin with unknown probability $p$ of heads can be flipped as often as desired. A Bernoulli factory for a function $f$ is an algorithm that uses flips of the coin together with auxiliary randomness to flip a single coin with probability $f(p)$ of heads. Applications include perfect sampling from the stationary distribution of certain regenerative processes. When $f$ is analytic, the problem can be reduced to a Bernoulli factory of the form $f(p)=C p$ for constant $C$. Presented here is a new algorithm that for small values of $C p$, requires roughly only $C$ coin flips. From information theoretic considerations, this is also conjectured to be (to first order) the minimum number of flips needed by any such algorithm.

For large values of $C p$, the new algorithm can also be used to build a new Bernoulli factory that uses only $80 \%$ of the expected coin flips of the older method. In addition, the new method also applies to the more general problem of a linear multivariate Bernoulli factory, where there are $k$ coins, the $k$ th coin has unknown probability $p_{k}$ of heads, and the goal is to simulate a coin flip with probability $C_{1} p_{1}+\cdots+C_{k} p_{k}$ of heads.
\end{abstract}

Keywords: randomized algorithm, near perfect simulation, regenerative processes

MSC Classes: 65C50, 68Q17

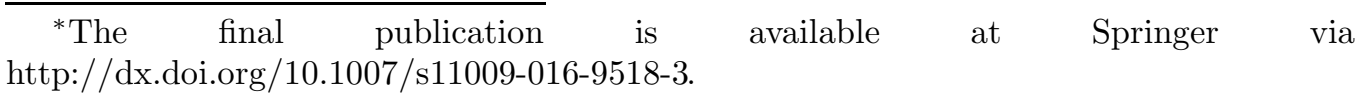




\section{Introduction}

The notion of a Bernoulli factory was introduced in Asmussen, Glynn, and Thorisson (1992) in the context of generating samples exactly from the stationary distribution of a regenerative Markov process. A Bernoulli factory works as follows. Suppose we have the ability to draw independent identically distributed (iid) Bernoulli random variables, each of which is 1 with probability $p$ and 0 with probability $1-p$ (write $X \sim \operatorname{Bern}(p)$.) Then given a function $f$, the goal is to use a random number of draws from $X$ to build a new random variable which is also Bernoulli, but with chance $f(p)$ of being 1 for a specified function $f$. In Asmussen, Glynn, and Thorisson (1992), the needed function was a linear function, namely a constant times $p$. This simple case generalizes: in Nacu and Peres (2005) it was shown that the ability to draw from $f(p)=2 p$ could be used to build a Bernoulli factory for any analytic $f$ that was bounded away from 1.

The focus here is on building a nearly optimal linear Bernoulli factory where $C p$ is known to be small. This is nearly optimal in the sense that it uses (to first order) only $C$ flips of the coin, and there is strong evidence to indicate that at least $C$ flips are necessary. As in Huber (to appear), a Bernoulli factory can be defined as follows.

Definition 1. Given $p^{*} \in(0,1]$ and a function $f:\left[0, p^{*}\right] \rightarrow[0,1]$, let $\mathcal{A}$ be a computable function that takes as input a number $u \in[0,1]$ together with a sequence of values in $\{0,1\}$, and returns an output in $\{0,1\}$. For any $p \in\left[0, p^{*}\right], X_{1}, X_{2}, \ldots$ iid $\operatorname{Bern}(p)$, and $U \sim \operatorname{Unif}([0,1])$, let $T$ be the infimum of times $t$ such that the value of $\mathcal{A}\left(U, X_{1}, X_{2}, \ldots\right)$ only depends on the values of $X_{1}, \ldots, X_{t}$. If the following holds, then call $\mathcal{A}$ a Bernoulli factory.

1. $T$ is a stopping time with respect to the natural filtration that is finite with probability 1.

2. $\mathcal{A}\left(U, X_{1}, X_{2}, \ldots\right) \sim \operatorname{Bern}(f(p))$.

Call $T$ the running time of the Bernoulli factory.

Colloquially, a draw $X \sim \operatorname{Bern}(p)$ will be refereed to as a coin flip, or more specifically, a $p$-coin flip. The result $X=1$ corresponds to heads on the coin, while $X=0$ indicates tails. So a Bernoulli factory attempts to flip a coin with $f(p)$ chance of heads, by using a random number of coin flips from the original coin together with some auxiliary randomness. 
Asmussen, Glynn, and Thorisson (1992) introduced Bernoulli factories for an application in perfect simulation, but did not show that they exist. Keane and O'Brien (1994) constructed the first general Bernoulli factories, showing that such a factory with finite running time existed if and only if $f(p)$ was continuous over $\left[0, p^{*}\right]$ for some $p^{*} \in(0,1]$, and either it holds that $f(p)$ is identically 0 or 1 , or that both $f(p)$ and $1-f(p)$ are polynomially bounded away from 0 and 1 over the allowable range of $p$.

Their strategy for building a Bernoulli factory was to construct Bernstein polynomials that approximated the function $f(p)$ as closely as possible. Bernstein polynomials are linear combinations of functions of the form $p^{k}(1-p)^{n-k}$ where both $n$ and $k \leq n$ are nonnegative integers. Such polynomials can be created from the coin by flipping it $n$ times and seeing if exactly $k$ heads and $n-k$ tails result. Keane and O'Brien could show that the running time $T$ was finite with probability 1 for their algorithm, but not much more. In particular, they could not show any bounds on the average running time, or even that it was finite.

Nacu and Peres (2005) developed this approach further, and showed that Bernstein polynomials could be constructed tightly enough that the running time would have a finite expectation. In addition, they showed that the tail of the distribution of their running time declined exponentially. Moreover, their work contained a proof that $f(p)=2 p$ is in a sense the most important function, since it can be used to construct a Bernoulli factory for any function that is both real analytic over $[0,1]$ and bounded away from 1 .

However, their approach was not a practical algorithm. While the number of coin flips had finite expectation, the amount of memory and time needed to compute the function $\mathcal{A}$ grew exponentially with the number of flips. Work of Łatuszyński, Kosmidis, Papspiliopoulos, and Roberts (2011) solved this issue, and gave the first practical implementation of the Nacu and Peres approach. Their approach created a pair of reverse time processes, one a supermartingale, the other a submartingale, that converged on the target $f(p)$. The values could be computed without the exponential overhead associated with the Nacu-Peres algorithm.

To bound $f(p)=C p$ away from 1 , they considered the function $f(p)=$ $\min \{C p, 1-\epsilon\}$ so that the function was defined over the entirety of $[0,1]$. However, this was not strictly necessary, as in the original application of Asmussen, Glynn, and Thorisson (1992), it was possible to easily insure that $f(p) \leq 1-\epsilon$. By not trying to sample from the function $f(p)=\min \{C p, 1-\epsilon\}$ for all values of $p$, but only for those with $C p \leq 1-\epsilon$, a new approach became 
possible.

This new approach was developed in Huber (to appear), and was the first that did not begin with Bernstein polynomial approximations. Instead, this approach used flips of the coin to alter the problem in ways that insured that the final output had the correct distribution. For instance, suppose the goal was to generate a coin with probability of heads $2 p$. Then flip the original coin once. If the coin is heads, the the output is heads. Otherwise, it is necessary to flip a $p /(1-p)$-coin.

That way, the chance the final output is heads is $p(1)+(1-p) \cdot p /(1-p)=$ $2 p$. By advancing carefully in this manner, it was shown how to build a Bernoulli factory such that for $C p \leq 1-\epsilon$ where $\epsilon$ is a known constant,

$$
\mathbb{E}[T] \leq 9.5 C \epsilon^{-1} \text {. }
$$

Moreover, the same work showed that this running time is the best possible up to a constant. Specifically, in Huber (to appear) it was shown that that any Bernoulli factory (to first order) must use on average at least $0.04 C \epsilon^{-1}$ coin flips. It remains an open question what the best constants for the lower and upper bounds are, although there is strong reason to believe (see Section 4) that on average at least $C$ flips (to first order) are necessary to generate a $C p$ coin.

The primary application of the Bernoulli factory, starting with Asmussen, Glynn, and Thorisson (1992), is to generate perfect samples from the stationary distribution of regenerative Markov chains. In Lee, Doucet and Łatusvzński (2014), it was shown how to use the Bernoulli factory in Huber (to appear) to generate coins where $C p \leq 2 / 3$. Under this condition, the older algorithm gave a bound of 38 coin flips on the average number needed.

Without going into the details of their algorithm, by tripling the expected running time of there algorithm, it is possible to ensure that $C p \leq 2 / 9$. Under these conditions, the expected number of flips for the new algorithm is bounded above by 7.8 (see Theorem 11) giving an algorithm that only requires $62 \%$ as many work on average as the old one.

This work gives the following results.

1. For $C p$ small, an algorithm will be given that uses only $C$ coin flips on average.

2. For $C p$ at most $1-\epsilon$ for known $\epsilon$, an algorithm will be given that uses only $7.57 C \epsilon^{-1}$ coin flips on average. 
3. The new algorithm can be extended from the function $C p$ for single variate coins to the multivariate coin problem where there are $k$ coins with unknown means $p_{1}, \ldots, p_{k}$. Suppose the goal is to generate $\operatorname{Bern}(r)$ where

$$
r\left(p_{1}, \ldots, p_{k}\right)=C_{1} p_{1}+\cdots+C_{k} p_{k} .
$$

Then setting $C=C_{1}+\cdots+C_{k}$, the algorithm in the multivariate case has running time equal to the single coin case.

More precisely, the running time of the new algorithm is given as follows.

Theorem 1. Suppose it is known that $C p \leq M$ for a constant $M<1 / 2$. Then there exists an algorithm for producing a $C p$-coin that uses on average at most

$$
\frac{C}{(1-2 M)(1+C p)}+C p \cdot\left[C \frac{15.2}{1-2 M+C p}\right]
$$

coin flips.

This theorem is shown in Section 3.5. The multivariate version is similar, and is shown in Section 5 .

Theorem 2. Let $C=C_{1}+\cdots+C_{k}$, and $r=C_{1} p_{1}+\cdots+C_{k} p_{k}$, Suppose it is known that $r \leq M$ for a constant $M<1 / 2$. Then there exists an algorithm for producing an $r$-coin that uses on average at most

$$
\frac{C}{(1-2 M)(1+r)}+C p \cdot\left[C \frac{15.2}{1-2 M+r}\right]
$$

flips from among the $k$ coins.

The remainder of this paper is organized as follows. Section 2 presents the algorithm for small $r$, and shows correctness and the bound on the running time. Section 3 gives the extension to the multivariate problem and for larger values of $r$, and also includes the proofs of correctness and the bound on the running time. Finally, Section 4 considers why $C$ flips is likely the best possible. 


\section{The algorithm for small $C p$}

Let $r=C p$. The first piece of the algorithm is a method for drawing from the logistic Bernoulli factory

$$
f(p)=\frac{r}{1+r}
$$

that uses $T$ coins, where $\mathbb{E}[T]=C /(1+r)$.

As usual, say that $X$ is exponential with rate $\lambda($ write $X \sim \operatorname{Exp}(\lambda)$ ) if $X$ has density $f_{X}(s)=\exp (-\lambda s) \mathbb{1}(s \geq 0)$. Here $\mathbb{1}(\cdot)$ is the indicator function that evaluates to 1 when the argument is true and 0 when the argument is false. The following basic facts about exponentials will prove useful.

Fact 1. Let $X \sim \operatorname{Exp}\left(\lambda_{1}\right)$ and $Y \sim \operatorname{Exp}\left(\lambda_{2}\right)$ be independent. Then $\mathbb{P}(X \leq$ $Y)=\lambda_{1} /\left(\lambda_{1}+\lambda_{2}\right)$.

Fact 2 (Memoryless). If $X \sim \operatorname{Exp}(\lambda)$, then for $s>0$, the conditional distribution of $X-s$ given $X>s$ is exponential with rate $\lambda$ as well. That is, $[X-s \mid X>s] \sim \operatorname{Exp}(\lambda)$.

Exponentials can be employed to define a one dimensional Poisson point process.

Definition 2. Let $A_{1}, A_{2}, \ldots$ be independent and identically distributed (iid) exponential random variables with rate $\lambda$. Then

$$
P=\left\{A_{1}, A_{1}+A_{2}, A_{1}+A_{2}+A_{3}, \ldots\right\}
$$

forms a Poisson point process on $[0, \infty)$ of rate $\lambda$. For $[a, b] \subset[0, \infty), P \cap[a, b]$ is a Poisson point process on $[a, b]$ of rate $\lambda$.

Several well known facts about Poisson point processes are useful.

Fact 3. The converse of the definition holds: any Poisson point process $P \subset[0, \infty)$ of rate $\lambda$ with points $0<P_{1}<P_{2}<\cdots$ has $P_{1} \sim \operatorname{Exp}(\lambda)$ and $P_{i}-P_{i-1} \sim \operatorname{Exp}(\lambda)$, and all these exponentials are independent.

Fact 4. Let $P=\left\{P_{1}, P_{2}, \ldots\right\}$ be a Poisson point process. Let $B_{1}, B_{2}, \ldots$ be a sequence of iid $\operatorname{Bern}(p)$ random variables. Then $P^{\prime}=\left\{P_{i}: B_{i}=1\right\}$ is a Poisson point process of rate $\lambda p$. [The process $P^{\prime}$ is called the thinned process.] 
Fact 5. Let $P_{1}$ and $P_{2}$ be independent Poisson point processes of rate $\lambda_{1}$ and $\lambda_{2}$ over $[0, \infty)$. Then $P_{1} \cup P_{2}$ is a Poisson point process of rate $\lambda_{1}+\lambda_{2}$ over $[0, \infty)$.

Fact 6. The expected number of points in a Poisson point process of rate $\lambda$ over $[a, b]$ is Poisson distributed with mean $\lambda(b-a)$.

These ideas can be used to build the logistic Bernoulli factory for $r /(1+r)$.

\begin{tabular}{l}
\hline Logistic_Bernoulli_Factory $\quad$ Input: $C$ \\
\hline 1) $X \leftarrow 0, \operatorname{draw} A \leftarrow \operatorname{Exp}(1)$ \\
2) $\quad$ Draw $T \leftarrow \operatorname{Exp}(C)$ \\
3) While $X=0$ and $T<A$ \\
4) $\quad$ Draw $B \leftarrow \operatorname{Bern}(p)$ \\
5) $\quad$ If $B=1$ then $X=1$, else $T \leftarrow T+\operatorname{Exp}(C)$ \\
$6) \quad$ Return $X$ \\
\hline
\end{tabular}

Note that line 4 can be accomplished in constant time (with $\Theta(k)$ preprocessing time) using the Alias method of Walker (1974).

Lemma 1. The output of Logistic_Bernoulli_Factory is a Bernoulli with mean $r /(1+r)$.

Proof. Let $T_{1}, T_{2}, \ldots$ be the successive values of $T$ taken on in the algorithm, and $B_{1}, B_{2}, \ldots$ the successive values of $B$. Since $T_{i+1}-T_{i}$ is $\operatorname{Exp}(C)$, the $\left\{T_{i}\right\}$ form a Poisson point process $P$ of rate $C$. Let $P^{\prime}$ be the points $T_{i} \in P$ with $B_{i}=1$. Then $P^{\prime}$ is a point process with rate $C p=r$.

Let $T_{1}^{\prime}=\min \left\{P^{\prime}\right\}$. The while loop examines the $P^{\prime}$ process, and returns 1 if $T_{1}^{\prime}<A$, and 0 otherwise. By Fact 3 , $T_{1}^{\prime} \sim \operatorname{Exp}(r)$, and $A \sim \operatorname{Exp}(1)$, so $\mathbb{P}\left(T_{1}^{\prime}<A\right)=r /(1+r)$ by Fact 1 .

Lemma 2. In one call to Logistic_Bernoulli_Factory, the expected number of coin flips needed is $C /(1+r)$.

Proof. The Poisson process of rate $C$ combined with the process of rate 1 forms a Poisson point process of rate $C+1$. The chance that any point of this process is from the thinned rate $C p$ process combined with the rate 1 process is $(C p+1) /(C+1)$. Therefore, the number of points generated in the rate $C+1$ process has a geometric distribution with mean $(C+1) /(C p+1)$. 
Each of these points has a $C /(C+1)$ chance of coming from the rate $C$ process initially, and so requires a coin flip. Therefore, combining these effects gives an expected number of coin flips of $[C /(C+1)][(C+1) /(C p+1)]=$ $C /(r+1)$.

Now suppose that there is a known $M<1 / 2$ such that $r \leq M$. Let $\mathrm{BF}(C)$ denote the Bernoulli Factory from Huber (to appear) that flips a $C p$ coin using on average $9.5 C(1-M)^{-1}$ flips of the original coin. Consider the following algorithm.

\begin{tabular}{ll}
\hline Small_r_1D_Bernoulli_Factory & Input: $\quad C, M$ \\
\hline 1) & $\beta \leftarrow 1 /(1-2 M)$ \\
2) & Draw $Y \leftarrow$ Logistic_Bernoulli_Factory $(\beta C)$ \\
$3)$ & Draw $B \leftarrow$ Bern $(1 / \beta)$ \\
4) & If $Y=0$, then $X \leftarrow 0$ \\
5) & Elseif $Y=1$ and $B=1$, then $X \leftarrow 1$ \\
$6)$ & Else $X \leftarrow \mathrm{BF}(\beta C /(\beta-1))$ \\
\hline
\end{tabular}

Lemma 3. Algorithm Small_r_1D_Bernoulli_Factory produces a Bernoulli distributed output with mean $C p \leq M<1 / 2$, and requires at most (on average)

$$
\frac{C}{(1-2 M)(1+C p)}+C p \cdot\left[19 C \frac{1}{1-2 M+C p}\right]
$$

coin flips to do so.

Note that for small $p$ and $M$, this running time is to first order just $C$.

Proof. First show correctness. Let $A_{1}$ be the event that $Y=1$ and $B=1$ in the algorithm (in which case line 5 sets $X$ to be 1 ), and $A_{2}$ be the event that $Y=1, B=0$, and a call to $\mathrm{BF}(C \beta /(\beta-1)$ ) returns a 1 (in which case line 7 sets $X$ to be 1). These are disjoint events, and the output of the algorithm is $X=\mathbb{1}\left(A_{1}\right)+\mathbb{1}\left(A_{2}\right)$. Therefore,

$$
\mathbb{P}(X=1)=\mathbb{P}\left(A_{1}\right)+\mathbb{P}\left(A_{2}\right) .
$$

The value of $Y$ is the call to Logistic_Bernoulli_Factory $(\beta C)$, and so $\mathbb{P}(Y=1)=\beta C p /(1+\beta C p)$. For the Bernoulli $B, \mathbb{P}(B=1)=1 / \beta$. Therefore $\mathbb{P}(A)=\mathbb{P}(Y=1) \mathbb{P}(B=1)=C p /(1+\beta C p)$. 
The output of $\operatorname{BF}(C \beta /(\beta-1))$ is 1 with probability equal to $\beta C p$, so

$$
\mathbb{P}\left(A_{2}\right)=\frac{\beta C p}{1+\beta C p}(1-1 / \beta) \frac{\beta C p}{\beta-1}=C p \frac{\beta C p}{1+\beta C p}
$$

Therefore

$$
\mathbb{P}(X=1)=C p \frac{1}{1+\beta C p}+C p \frac{\beta C p}{1+\beta C p}=C p
$$

as desired.

Now for the running time. Lemma 2 gives a running time of $\beta C /(1+C p)$ for line 1 . Line 5 is executed with probability $(\beta-1) C p /(1+\beta C p)$. By the way $\beta$ was chosen, $C p \beta /(\beta-1) \leq 1 / 2$. Therefore, Theorem 1.1 of Huber (to appear) gives that the call to $\mathrm{BF}(C \beta /(\beta-1))$ requires at most $19[C \beta /(\beta-$ 1)] flips. Therefore, the total number of flips is on average at most

$$
\frac{C}{(1-2 M)(1+C p)}+C p \cdot\left[\frac{19 C}{1-2 M+C p}\right] .
$$

This is close to Theorem 1, but the constant of 19 in the second term is larger. To improve this algorithm, and eliminate the need for the call to the old $\mathrm{BF}$ algorithm, it is necessary to consider what happens for larger $r$.

\section{Large $r$ algorithm}

In this section, the algorithm of the previous section is improved to allow for all $r \in[0,1-\epsilon]$, where $\epsilon$ is arbitrarily close to 0 . Along the way, the older 9.5C $\epsilon^{-1}$ algorithm of Huber (to appear) is improved to a $7.5 C \epsilon^{-1}$ algorithm.

The first step is to build a random coin flip whose mean is slightly larger than $r$. If this coin is tails, return tails for $r$. If the coin returns heads, heads will be returned with probability close to 1 . Otherwise, a new coin will need to be flipped.

\subsection{A coin flip with mean slightly larger than $r$}

Consider an asymmetric random walk on the integers $\Omega=\{0,1, \ldots, m\}$, where given the current state $X_{t}$, the next state is either $\max \left\{0, X_{t}-1\right\}$, or $\min \left\{X_{t}+1, m\right\}$. The transition probabilities are

$\mathbb{P}\left(X_{t+1}=\min \{i+1, m\} \mid X_{t}=i\right)=p_{r}, \mathbb{P}\left(X_{t+1}=\max \{i-1,0\} \mid X_{t}=i\right)=q_{r}$, 
where $p_{r}+q_{r}=1$. This is also called the Gambler's Ruin walk.

The following facts about this well known process will be helpful.

Fact 7. Suppose $p_{r} \neq q_{r}$ and $T=\inf \left\{t: X_{t} \in\{0, m\}\right\}$. Then

$$
\begin{aligned}
\mathbb{P}\left(X_{T}=m\right) & =\frac{1-\left(q_{r} / p_{r}\right)^{X_{0}}}{1-\left(q_{r} / p_{r}\right)^{m}} \\
\mathbb{E}[T] & =\frac{X_{0}}{q_{r}-p_{r}}-\frac{m}{q_{r}-p_{r}} \cdot \mathbb{P}\left(X_{T}=m\right) .
\end{aligned}
$$

Fact 8. Suppose $X_{0}=m, p_{r}<q_{r}$, and $T=\inf \left\{t: X_{t}=0\right\}$. Then $\mathbb{E}[T] \leq m /\left(q_{r}-p_{r}\right)$.

Consider the following Bernoulli factory that begins a Gambler's Ruin walk starting at state 1 , and returns heads if the state reaches 0 before it reaches $m$.

\begin{tabular}{ll}
\hline A & Input: $m, C$ \\
\hline 1) & $s \leftarrow 1$ \\
2) & While $s \in\{1,2, \ldots, m-1\}$ \\
3) & $B \leftarrow$ Logistic_Bernoulli_Factory $(C)$ \\
4) & $s \leftarrow s-2 B+1$ \\
5) & Return $\mathbb{1}(s=0)$ \\
\hline
\end{tabular}

Lemma 4. The output of $\mathrm{A}$ is a Bernoulli with mean $r\left(1-r^{m-1}\right) /\left(1-r^{m}\right)$.

Proof. Logistic_Bernoulli_Factory outputs a Bernoulli that has mean $r /(1+r)$. Hence $p_{r}=1 /(1+r), q_{r}=r /(1+r)$, and $q_{r} / p_{r}=r$. From Fact 7 , in line 5 that makes $\mathbb{P}(I=0)=1-(1-r) /\left(1-r^{m}\right)=\left(r-r^{m}\right) /\left(1-r^{m}\right)=$ $r\left(1-r^{m-1}\right) /\left(1-r^{m}\right)$.

Lemma 5. The expected number of coin flips used by $\mathrm{A}$ is at most $C(m-1)$.

Proof. As in the last proof $p_{r}=1 /(1+r)$ and $q_{r}=r /(1+r)$. So

$$
q_{r}-p_{r}=\frac{r}{1+r}-\frac{1}{1+r}=-\frac{1-r}{1+r},
$$

and $\left(q_{r} / p_{r}\right)=r$. Using (3),

$$
\mathbb{E}[T]=\frac{1+r}{1-r} \cdot\left[m \frac{1-r}{1-r^{m}}-1\right]=(1+r)\left[\frac{m}{1-r^{m}}-\frac{1}{1-r}\right] .
$$


Let $f(r)=m /\left(1-r^{m}\right)-1 /(1-r)$. Then it holds that $f(r)<m-1$ for all $r \in(0,1)$ and $m \geq 1$. Note that for all $r \in(0,1)$ :

$$
\begin{aligned}
f(r)<m-1 & \Leftrightarrow m(1-r)-\frac{\left(1-r^{m}\right)}{1-r}<(m-1)\left(1-r^{m}\right) \\
& \Leftrightarrow m-m r-1-r-\cdots-r^{m-1}<m-1-r^{m}(m-1) \\
& \Leftrightarrow(m-1) r^{m}<r+r^{2}+\cdots+r^{m-1}+m r \\
& \Leftrightarrow m-1<r^{1-m}+r^{2-m}+\cdots+r^{-1}+m r^{1-m} .
\end{aligned}
$$

Since $r \in(0,1), r^{i-m} \geq 1$ for all $i \in\{1, \ldots, m-1\}$, so the right hand side is strictly greater than the left hand side. Note $f(0)=m-1$, so for $r \in[0,1-\epsilon]$, the function is at most $m-1$.

Each call to line 3 requires on average $C /(1+r)$ time by Lemma 2 . Together, the overall number of steps (on average) is at most

$$
(1+r)(m-1) \frac{C}{1+r}=C(m-1)
$$

\subsection{After the flip}

Here is how A can be useful. Using $A$, it is possible to generate a Bernoulli random variable that is 1 with probability

$$
p_{\beta}=\beta r \frac{1-(\beta r)^{m-1}}{1-(\beta r)^{m}}
$$

for any constant $\beta>1$. By choosing $\beta$ large enough, $p_{\beta} \geq r$. Note that $p_{\beta} / \beta \leq r$. So $r \in\left[p_{\beta} / \beta, p_{\beta}\right]$.

So the algorithm works as follows. First flip a $p_{\beta}$-coin. If it is tails, then return tails for the $r$-coin as well. If it is heads, then flip a $(1 / \beta)$-coin. If that is heads as well, return heads for the $r$-coin. Otherwise, flip a $p^{\prime}$-coin, and return the same value for the $r$-coin.

For this algorithm to work, $p^{\prime}$ must satisfy:

$$
r=\frac{p_{\beta}}{\beta}+p^{\prime} p_{\beta}(1-(1 / \beta)) .
$$

Solving for $p^{\prime}$ gives

$$
p^{\prime}=\frac{1}{\beta-1}\left[\frac{(\beta r)^{m-1}}{1+(\beta r)^{1}+\cdots+(\beta r)^{m-2}}\right],
$$

so the next step of the algorithm is figuring out how to generate a $p^{\prime}$-coin. 


\subsection{Generating a $p^{\prime}$-coin}

Fortunately, we do not have to actually generate a $p^{\prime}$-coin for all possible values of $\beta$, as we are allowed to choose the value of $\beta$ to use, as long as $p_{\beta} \geq r$ for our choice of $\beta$. Let

$$
\beta=1+\frac{1}{m-1},
$$

so $(\beta-1)^{-1}=m-1$. Then

$$
p^{\prime}=\frac{(m-1)(\beta r)^{m-1}}{1+(\beta r)+\cdots+(\beta r)^{m-2}} .
$$

Note that $p^{\prime} \leq 1$ which gives that $p_{\beta} \geq r$ for this choice of $\beta$.

The algorithm for generating a $p^{\prime}$-coin for $p^{\prime}$ as in (4) will be called B here, and is shown graphically in Figure 1. Notice that if the first flip is heads and the second flip is tails, then our problem has changed to the same problem, but with $m$ reduced to $m-1$.

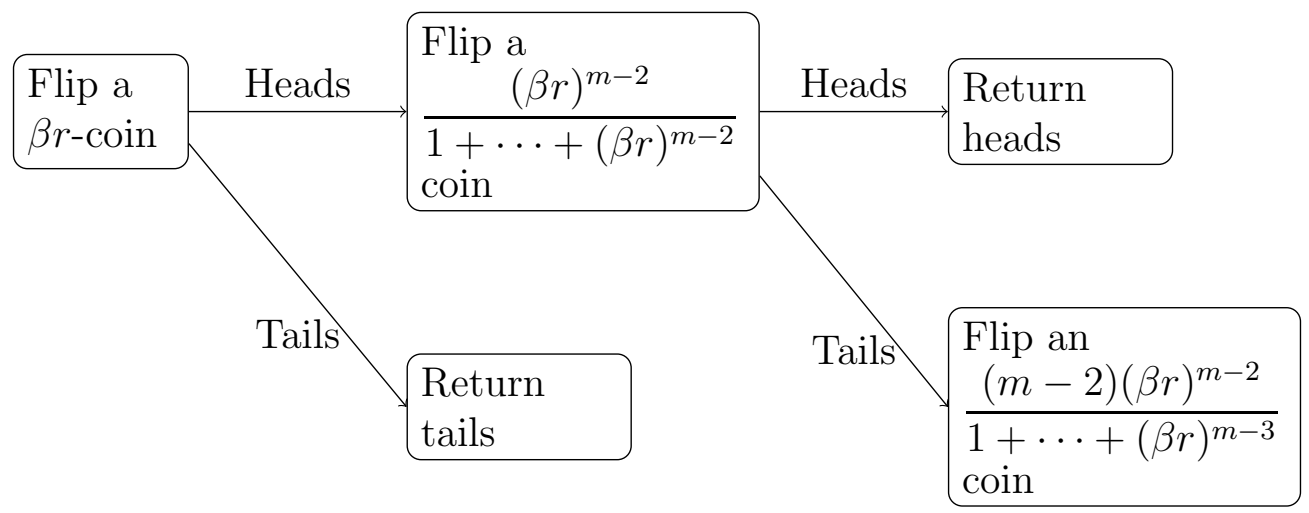

Figure 1: A graphical illustration of Algorithm B.

To utilize this procedure, it is necessary to be able to generate a $(\beta r)^{m-2} /(1+$ $\cdots+(\beta r)^{m-2}$ coin. Fortunately, this can be accomplished fairly quickly using the Gambler's ruin chain from earlier. 
High_Power_Logistic_BF

Input: $\quad m, \beta, C$ Output: $\quad X \sim \operatorname{Bern}\left((\beta r)^{m} /\left(1+\cdots+(\beta r)^{m}\right)\right)$

1) $s \leftarrow 1$

2) While $s \in\{1, \ldots, m\}$

3) $\quad$ Draw $B \leftarrow$ Logistic_Bernoulli_Factory $(\beta C)$

4) $s \leftarrow s+2 B-1$

5) $X \leftarrow \mathbb{1}(s=m+1)$

Lemma 6. The output of High_Power_Logistic_BF has distribution Bern $\left((\beta r)^{m} /(1+\right.$ $\left.\left.\cdots+(\beta r)^{m}\right)\right)$. The expected number of coin flips used is at most $\beta C /(1-\beta r)$.

Proof. This is a Gambler's ruin where $p=\beta r /(1+\beta r)$ and $q=1 /(1+\beta r)$, so $q / p=1 /(\beta r)$. Hence from Fact 7 ,

$$
\mathbb{P}(s=m+1)=\frac{1-(1 /(\beta r))^{1}}{1-(1 /(\beta r))^{m+1}}=\frac{(\beta r)^{m}(1-\beta r)}{1-(\beta r)^{m+1}}=\frac{(\beta r)^{m}}{1+\cdots+(\beta r)^{m}} .
$$

Also from Fact [7, note $q-p=(1+\beta r) /(1-\beta r)$, so if $T$ is the number of times line 3 is called,

$$
\mathbb{E}[T]=\frac{1+\beta r}{1-\beta r}\left[1-(m+1) \frac{(\beta r)^{m}(1-\beta r)}{1-(\beta r)^{m+1}}\right] \leq \frac{1+\beta r}{1-\beta r} .
$$

Each call to Logistic_Bernoulli_Factory takes time $\beta C /(1+\beta r)$, so the overall number of coin flips (on average) is at most $\beta C /(1-\beta r)$.

In pseudocode, algorithm B looks like this.

\begin{tabular}{lc}
\hline B & Input: $\epsilon, m, \beta, C \quad$ Output: $X$ \\
\hline 1$)$ & $X \leftarrow 0.5$ \\
$2)$ & While $X \notin\{0,1\}$ \\
$3)$ & Draw $B_{1} \leftarrow$ Linear_Bernoulli_Factory $(1-(1-\epsilon) \beta, \beta \cdot C)$ \\
$4)$ & If $B_{1}=0$ then $X \leftarrow 0$ \\
$5)$ & Else \\
$6)$ & $B_{2} \leftarrow$ High_Power_Logistic_BF $(m-2, \beta, C)$ \\
$7)$ & If $B_{2}=1$ then $X \leftarrow 1$ \\
$8)$ & Else $m \leftarrow m-1$ \\
\hline
\end{tabular}


The most important thing to note here is that like many perfect simulation algorithms, this method employs recursion. We do not yet have an algorithm for completing line 3! However, this algorithm B can be used as a subroutine to create such an algorithm, and then this subroutine will call the finished algorithm.

Lemma 7. The output of B has distribution

$$
\operatorname{Bern}\left((m-1)(\beta r)^{m-1} /\left(1+\cdots+(\beta r)^{m-2}\right)\right) .
$$

Proof. The proof is by induction. When $m=2$, if $B_{1}=1$ then $B_{2} \sim \operatorname{Bern}(1)$, so $X=1$ with probability $\beta r$ as desired.

Now suppose that the result holds for $m$, consider $m+1$. Then

$$
\begin{aligned}
\mathbb{P}(X=1) & =\beta r\left[\frac{(\beta r)^{m-2}}{1+\cdots+(\beta r)^{m-2}}+\frac{1+\cdots+(\beta r)^{m-3}}{1+\cdots+(\beta r)^{m-2}} \cdot \frac{(m-2)(\beta r)^{m-2}}{1+\cdots+(\beta r)^{m-3}}\right] \\
& =\frac{(m-1)(\beta r)^{m-1}}{1+\cdots+(\beta r)^{m-2}},
\end{aligned}
$$

completing the induction.

\subsection{The new linear Bernoulli Factory}

With these preliminaries in place, the overall algorithm is as follows.

\begin{tabular}{ll}
\hline Linear_Bernoulli_Factory $\quad$ Input: $\epsilon, C$ & Output: $B$ \\
\hline 1) & $m \leftarrow\left\lceil 4.5 \epsilon^{-1}\right\rceil+1, \beta \leftarrow 1+1 /(m-1)$ \\
2) & $B_{1} \leftarrow \mathrm{A}(m, \beta \cdot C)$ \\
3) & If $B_{1}=1$ \\
4) & Draw $B_{2} \leftarrow$ Bern $(1 / \beta)$ \\
5) & If $B_{2}=1$ then $B \leftarrow 1$ \\
$6)$ & Else \\
7) & Draw $B \leftarrow \mathrm{B}(m, \beta, C)$ \\
$8)$ & Else $B \leftarrow 0$ \\
\hline
\end{tabular}

Now consider the expected number of coin flips used by the algorithm. As will become clear in the proofs of Lemmas 8 and 10 below, making $m=$ $\Theta\left(\epsilon^{-1}\right)$ is the correct choice. That leaves the choice of constant up to us, and 
the constant of 4.5 from Line 1 was chosen to make the overall running time as small as possible.

This algorithm calls A and B. Line 2 of B needs to draw a Bern $(\beta r)$ random variable. The best way to draw these random variables is to recursively call Linear_Bernoulli_Factory. In order to ensure that this back in forth calling eventually comes to a halt with probability 1 , it is easiest to bound the total expected number of calls to Linear_Bernoulli_Factory.

Lemma 8. The expected number of calls to Linear_Bernoulli_Factory is at most 1.4 .

Proof. Let $m_{1}$ be the value of $m$ in the first call to Linear_Bernoulli_Factory, and $\beta_{1}=1+1 /\left(m_{1}-1\right)$. From this first call there is a chance of calling $\mathrm{B}$, which in turn calls Linear_Bernoulli_Factory with $m_{2}$ and $\beta_{2}$. Each of those second generation calls might call a third generation, and so on. To bound the expected number of calls to Linear_Bernoulli_Factory sum over all possible calls of the probability that that call is executed. Let $N_{i}$ denote the number of $i$ th generation calls.

The expected number of calls in the first generation is 1 . Consider a call in the second generation. In order for that call to be made, there must have been a call to $B$ from the first generation, and all prior second generation calls from line 3 of $\mathrm{B}$ must have had $B_{1}=0$. The number of times the while loop in $B$ is executed is stochastically dominated by a geometric random variable with mean $1 /\left(1-\beta_{1} r\right)$. Since

$$
\begin{aligned}
1-\beta_{1} r & \geq 1-\left(1+1 /\left\lceil 4.5 \epsilon^{-1}\right\rceil\right)(1-\epsilon) \\
& =(7 / 9) \epsilon+(2 / 9) \epsilon^{2},
\end{aligned}
$$

the number of calls made is bounded (in expectation) by $(9 / 7) \epsilon^{-1}$.

But before $\mathrm{B}$ is even called, first it must have held that $B_{1}=1$ and $B_{2}=0$ in lines 2 and 4 of the first generation call to Linear_Bernoulli_Factory.

The probability that a call to $\mathrm{B}$ is made is at most

$$
\left(1-1 / \beta_{1}\right) \beta r\left(1-\left(\beta_{1} r\right)^{m-1}\right) /\left(1-\left(\beta_{1} r\right)^{m}\right) \leq \frac{1}{m-1}
$$

So the expected number of calls to Linear_Bernoulli_Factory in the second generation is bounded by

$$
\mathbb{E}\left[N_{2} \mid N_{1}\right] \leq N_{1}\left[1 /\left(m_{1}-1\right)\right](9 / 7) \epsilon^{-1} \leq(2 / 7) N_{1} .
$$


This step forms the basis of an induction that gives $\mathbb{E}\left[N_{i}\right] \leq(2 / 7)^{i} N_{1}=$ $(2 / 7)^{i}$. Therefore $\sum \mathbb{E}\left[N_{i}\right] \leq 1 /(1-2 / 7)=1.4$.

Lemma 9. The output $B$ of Linear_Bernoulli_Factory has $B \sim \operatorname{Bern}(r)$.

Proof. Line 2 of B requires a draw $B_{1} \leftarrow \operatorname{Bern}(\beta r)$. Suppose that for the first $L$ times this line is called, the Linear_Bernoulli_Factory is called to generate this random variable. Then, from the $L+1$ st time onwards, an oracle generates the random variable.

We show by strong induction that Linear_Bernoulli_Factory generates from $\operatorname{Bern}(r)$ for any finite $M$. The base case when $M=0$ operates as follows. Lemma 7 immediately gives in this case that a call to $B$ returns a random

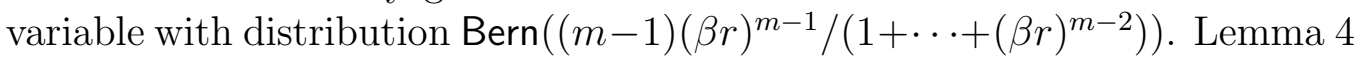
gives that $B_{1}$ from line 2 has distribution $\operatorname{Bern}\left((\beta r)^{m} /\left(1+\cdots+(\beta r)^{m}\right)\right)$. Putting this together gives

$$
\begin{aligned}
\mathbb{P}(B=1) & =(\beta r) \frac{1-(\beta r)^{m-1}}{1-(\beta r)^{m}}\left[\frac{1}{\beta}+\left(1-\frac{1}{\beta}\right) \frac{(m-1)(\beta r)^{m-1}}{1+\cdots+(\beta r)^{m-2}}\right] \\
& =(\beta r) \frac{1-(\beta r)^{m-1}}{1-(\beta r)^{m}}\left[\frac{1}{\beta}+\frac{1}{\beta} \frac{(\beta r)^{m-1}}{1+\cdots+(\beta r)^{m-2}}\right] \\
& =r \cdot \frac{\left(1-(\beta r)^{m-1}\right)}{1-(\beta r)^{m}} \cdot \frac{1+\cdots+(\beta r)^{m-1}}{1+\cdots+(\beta r)^{m-2}} \\
& =r .
\end{aligned}
$$

This is the rare induction proof where the base case is just as hard as the induction step. Suppose it holds for $L$, and consider what happens for call limit $L+1$. Then the first call to Linear_Bernoulli_Factory might call B, which might call Linear_Bernoulli_Factory. But the first such call has used up one call, so only has $L+1-1$ calls remaining, so by strong induction each returns the correct distribution. Hence Lemma 7 holds, and the first call returns the correct distribution by the same argument as the base case.

Let $N$ be the random number of calls to Linear_Bernoulli_Factory needed by the algorithm. Then let $B$ be the output when $N$ is unbounded, and $B_{M}$ be the output when a limit on calls equal to $L$ is in place. Then

$$
\begin{aligned}
\mathbb{P}(B=1) & =\mathbb{P}(B=1, N \leq L)+\mathbb{P}(B=1, N>L) \\
& =\mathbb{P}\left(B_{L}=1, N \leq M\right)+\mathbb{P}(B=1, N>L) \\
& =\mathbb{P}\left(B_{L}=1\right)-\mathbb{P}\left(B_{L}=1, N>L\right)+\mathbb{P}(B=1, N>L) .
\end{aligned}
$$


Both $\mathbb{P}\left(B_{L}, N>L\right)$ and $\mathbb{P}(B=1, N>L)$ are bounded above by $\mathbb{P}(N>L)$. Since by the last lemma $\mathbb{E}[N] \leq 1.4, \lim _{L \rightarrow \infty} \mathbb{P}(N>L)=0$. The only way this can hold for all $L$ is if $\mathbb{P}(B=1)=\mathbb{P}\left(B_{L}=1\right)$ for all $L$, so $B$ has the correct distribution.

Lemma 10. Linear_Bernoulli_Factory uses on average at most $7.67 C \epsilon^{-1}$ coin flips to generate $B \sim \operatorname{Bern}(r)$.

Proof. From the proof of Lemma 8 , the expected number of calls to the $i$ th generation of Linear_Bernoulli_Factory is bounded above by $(2 / 7)^{i}$.

From (6), at each successive generation of calls, $\epsilon$ is being multiplied by a factor of at least $7 / 9$. So an $i$ th generation call to Linear_Bernoulli_Factory has an $m$ value of at most $\left\lceil 4.5(9 / 7)^{i} \epsilon^{-1}\right\rceil+1$, where $\epsilon$ was the input for the 0th generation.

Coin flips occur during the call to A, and Lemma 4 bounds the expected number of coin flips by $C\left\lceil 4.5(9 / 7)^{i} \epsilon^{-1}\right\rceil$. So the expected total flips coming from the $i$ th generation of Linear_Bernoulli_Factory is at most $C\left[4.5(18 / 49)^{i} \epsilon^{-1}+(2 / 7)^{i}\right]$.

Now look at the flips coming from an $i$ th generation call to B. This generation is only called from an $i$ th generation call to Linear_Bernoulli_Factory, of which there the expected number is at most $(2 / 7)^{i}$. The call to B occurs with probability at most $(\beta-1) r$, so at most $r / m$. The while loop inside $B$ is run (on average) at most $\epsilon^{-1}$ times, each of which could make a call to High_Power_Logistic_BF. By Lemma 6 this requires at most $\beta C \epsilon^{-1}$ coin flips. So the total number of coin flips from an $i$ th generation call to $\mathrm{B}$ is at most

$$
(2 / 7)^{i}[r / m]\left[\beta C \epsilon^{-1}(9 / 7)^{i+1}\right] \epsilon^{-1} \leq(9 / 7) 4.5^{-1}(18 / 49)^{i} C \epsilon^{-1} .
$$

Summing over these flips and the ones from Linear_Bernoulli_Factory gives a total sum of

$$
(469 / 62) C \epsilon^{-1} \leq 7.57 C \epsilon^{-1}
$$

coin flips on average.

\subsection{Small $r$}

Now that a recursive algorithm for large $r$ has been built, a recursive analogue for Small_r_1D_Bernoulli_Factory works as follows. 


\begin{tabular}{ll}
\hline Small_r_Bernoulli_Factory $\quad$ Input: $\quad C, M$ \\
\hline 1) & $\beta \leftarrow 1 /(1-2 M)$ \\
2) & Draw $Y \leftarrow$ Logistic_Bernoulli_Factory $(\beta C)$ \\
3) & Draw $B \leftarrow$ Bern $(1 / \beta)$ \\
4) & If $Y=0$, then $X \leftarrow 0$ \\
5) & Elseif $Y=1$ and $B=1$, then $X \leftarrow 1$ \\
$6)$ & Else $X \leftarrow$ Linear_Bernoulli_Factory $(C \beta /(\beta-1))$ \\
\hline
\end{tabular}

Lemma 11. Algorithm Small_r_Bernoulli_Factory produces a Bernoulli distributed output with mean $C p \leq M<1 / 2$, and requires at most (on average)

$$
\frac{C}{(1-2 M)(1+C p)}+C p \cdot\left[C \frac{15.2}{1-2 M+C p}\right]
$$

coin flips to do so.

Proof. The proof is essentially the same as that of Lemma 3 ,

\section{Lower bound}

To see why it is unlikely that a method that uses fewer than $C \epsilon^{-1}$ coin flips can be constructed, consider building an unbiased estimate of $p$.

The standard estimate is to generate $X_{1}, \ldots, X_{n}$ iid $\operatorname{Bern}(p)$, and then use the sample average $\hat{p}_{n}=\left(X_{1}+\cdots+X_{n}\right) / n$ as an unbiased estimate of $p$. This estimate is unbiased, and has variance $p(1-p) / n$.

Now consider the estimate $Y / C$, where $Y \sim \operatorname{Bern}(C p)$. Then $\mathbb{E}[Y / C]=$ $C p / C=p$ so this estimate is also unbiased, and the variance is $C p(1-$ $C p) / C^{2}=p(1-C p) / C$. Therefore, this estimate that used one draw from $\operatorname{Bern}(C p)$ has the variance of the estimate that used $n=C(1-p) /(1-C p)$ draws from the $p$-coin.

The Cramér-Rao lower bound (see, for instance Bickel and Doksum (1977)) on the variance of an unbiased estimate of $p$ is

$$
\frac{p(1-p)}{n} \text {. }
$$

That is, any unbiased estimate that uses up to $n$ flips of the $p$-coin must have variance at least $p(1-p) / n$. That immediately gives that any algorithm for generating a $C p$-coin that uses a deterministic number $n$ of coin flips must 
have $n \geq C(1-p) /(1-C p)$. Of course, this does not quite apply to a Bernoulli Factory, because here a random number of coin flips is used.

However, it is strong evidence that $C(1-p) /(1-C p)$ is a lower bound on the expected number of coin flips needed by an algorithm.

\section{Multivariate Bernoulli Factory}

This new algorithm was designed for the single coin problem, in this section consider generating a coin flip whose probability of heads is the sum of the probability of heads on two different coins each of which has an unknown probability of heads. Unlike the single coin flip, there is no immediate application, however, it is useful to know that the single coin algorithm can be easily generalized to solve this problem should the need arise.

More generally, the goal is now to generate a coin flip with probability

$$
r=C_{1} p_{1}+\cdots+C_{k} p_{k}
$$

of heads, where $r$ is bounded away from 1, using as few flips of the coins as possible. When $k=1$, this is the linear Bernoulli factory studied in the previous sections. Formally, a multivariate Bernoulli factory is defined as follows.

Definition 3. Given a computable function $f:[0,1]^{k} \rightarrow[0,1]$, a multivariate Bernoulli factory is a computable function

$$
\mathcal{A}:[0,1] \times\left(\{0,1\}^{\{1,2, \ldots\}}\right)^{k} \rightarrow\{0,1\}
$$

such that if $U \sim \operatorname{Unif}([0,1])$ and the $X_{i, j}$ are independent random variables with $(\forall i \in\{1, \ldots, k\})(\forall j \in\{1,2, \ldots\})\left(X_{i, j} \sim X_{i}\right)$, then the following properties hold.

1. There exist random variables $\left(T_{1}, \ldots, T_{k}\right) \in\{1,2, \ldots\}^{k}$ such that the value of $\mathcal{A}\left(U,\left\{X_{1, i}\right\}_{i=1}^{\infty}, \ldots,\left\{X_{k, i}\right\}_{i=1}^{\infty}\right)$ only depends on the values of $\left\{X_{1, i}\right\}_{i=1}^{T_{1}}, \ldots,\left\{X_{k, i}\right\}_{i=1}^{T_{k}}$, and for all $\left(t_{1}, \ldots, t_{k}\right)$, the event $\left(T_{1}, \ldots, T_{k}\right)=$ $\left(t_{1}, \ldots, t_{k}\right)$ is measurable with respect to $\left\{X_{1, i}\right\}_{i=1}^{t_{1}}, \ldots,\left\{X_{k, i}\right\}_{i=1}^{t_{k}}$.

2. $\mathcal{A}\left(U,\left\{X_{1, i}\right\}_{i=1}^{\infty}, \ldots,\left\{X_{k, i}\right\}_{i=1}^{\infty}\right) \sim \operatorname{Bern}\left(f\left(p_{1}, p_{2}, \ldots, p_{k}\right)\right)$.

Call $T_{1}+\cdots+T_{k}$ the running time of the algorithm. 
The key to the single coin algorithm was generating a random variable that was exponential with rate parameter $r$. In the single coin case, this was done by generating a Poisson process of rate $C$, then thinning.

For the multivariate coin case, consider generating $k$ independent Poisson point processes $P_{1}, \ldots, P_{k}$, where $P_{i}$ has rate $C_{i}$. Then thin each process $P_{i}$ with coin $i$ to obtain a Poisson point process of rate $C_{i} p_{i}$. The union of these is a new Poisson point process of rate $r$, and the rest of the algorithm operates as before. The proofs of all the lemmas and Theorem 2 then proceeds in exactly the same way as given earlier.

\section{Acknowledgments}

This research supported by NSF DMS-1418495.

\section{References}

Asmussen S, Glynn PW, and Thorisson H (1992) Stationarity detection in the initial transient problem. ACM Trans Modeling and Computer Simulation $2(2): 130-157$.

Bickel PJ and Doksum KA (1977) Mathematical Statistics. Prentice Hall, New Jersey.

Huber M (to appear). Nearly optimal Bernoulli factories for linear functions. Combin Probab Comput, arXiv:1308.1562.

Keane MS and O'Brien GL (1994) A Bernoulli factory. ACM Trans Modeling and Computer Simulation 4:213-219.

Łatuszyński K, Kosmidis I, Papspiliopoulos O, and Roberts G (2011) Simulating events of unknown probabilities via reverse time martingales. Random Structures Algorithms 38(4):441-452.

Lee A, Doucet A, and Eatuszyński K (2014) Perfect simulation using atomic regeneration with application to Sequential Monte Carlo. arXiv: $1407.5770 \mathrm{v} 1$.

Nacu S and Peres Y (2005) Fast simulation of new coins from old. Ann Appl Probab 15(1A):93-115. 
Walker AJ (1974) New fast method for generating discrete random numbers with arbitrary frequency distributions. Electronics Letters 10(8):127. 